\title{
Restoration of calf deformities in a 35-year-old man following 2 calf reduction surgical procedures
}

\author{
Young-Shin Cho, MD \\ Young Clinic, Seoul, Rep. of Korea
}

\begin{abstract}
Calf surgery for slender and proportionate calves is currently being performed not only in women but also in Korean men. Calf liposuction or calf muscle resection can cause several adverse effects including contour deformities, adhesions, and contraction and shortening of muscles, among others. This case report describes reconstructive surgery for a calf deformity and complications of compensatory calf muscle hypertrophy after 2 muscle resection surgeries performed in a 35-year-old man. In this case, muscle resection-induced compensatory muscle hypertrophy can be treated by a selective nerve block. Adhesion of skin to muscle fascia and dermal dimples can be treated by fat transplantation with stem cells and injecting stem cells directly into the muscle layer.
\end{abstract}

Keywords: calf deformities; calf fat grafting; calf muscle resection surgery; calf restoration; calf selective nerve blocks; calf surgery complications

\section{Introduction}

The calf is the posterior part of the lower leg that made of fat and muscle. Over the last decade, large numbers of Korean women have been undergoing cosmetic/plastic surgery for slender and proportionate calves. Calf surgeries are primarily categorized as calf liposuction and calf muscle reduction operations. Calf muscle reduction surgery reduces the size of calf muscles through a nerve block procedure that selectively identifies and blocks the nerves that innervate the gastrocnemius and the lateral portion of the soleus muscles. This study reports a procedure that is being performed since 2007. Selective nerve blocks are performed, whereby weak electrical stimulation identifies and coagulates specific nerves to a radius of within 5 $\mathrm{mm}$ at $95^{\circ} \mathrm{C}$ using high frequency waves. In recent years, treatments are available to manage the adverse effects associated with calf reduction surgery; therefore, calf surgeries are being widely performed. Moreover, not only women but a growing number of men too are undergoing cosmetic surgery including calf reduction surgery. This paper describes a restorative procedure performed in a patient with a calf deformity who developed contraction and hypertrophy of the calf muscles after undergoing calf reduction (calf muscle resection surgery).

\section{Case report}

\section{Patient's history}

A 35-year-old man was treated for calf muscle hypertrophy. He underwent 3 calf nerve block procedures at 6-month intervals in 2015. However, the patient was dissatisfied with the surgical outcomes. In 2016, he underwent gastrocnemius muscle resection surgery twice. However, postoperatively, his right heel did not touch the ground secondary to a contour deformity and hypertrophy of both calves and contraction and shortening of

Received December 10, 2018; Accepted December 12, 2018

Corresponding author: Young-Shin Cho

E-mail: youngclinic@naver.com, ORCID: https://orcid.org/0000-0001-5423-2489

This is an Open Access article distributed under the terms of the Creative Commons Attribution Non-Commercial License (http://creativecommons.org/licenses/by-nc/4.0), which permits unrestricted non-commercial use, distribution, and reproduction in any medium, provided the original work is properly cited.

Copyright (c) 2018 Korean Society of Korean Cosmetic Surgery and Medicine (KSKCS \& KCCS). 
the muscles of the right calf that occurred as an adverse effect of the surgery. He reported discomfort particularly with going up and down stairs.

\section{Treatment method: case summary}

Pre-treatment state: gastrocnemius muscle contraction occurred after gastrocnemius muscle resection surgery, and a dimple deformity was observed affecting the patient's calves. Compensatory muscle hypertrophy was accompanied by hypertrophy of the soleus muscle, and the right heel did not touch the ground because of severe muscle contraction. Therefore, the patient complained of discomfort during walking. Gastrocnemius muscle resection surgery caused muscle contraction and a dimple deformity around the muscle. Compensatory muscular hypertrophy was observed in the upper layer of the medial gastrocnemius.

\section{Treatment}

During this study, $20 \mathrm{ml}$ of blood was obtained from the patient twice at 4 -week intervals to produce $2 \mathrm{ml}$ of platelet-rich plasma for subcutaneous and intramuscular injections. Two weeks after the second procedure, soleus and medial gastrocnemius nerve blocks were performed on the right and left calf, respectively.

After 4 weeks, $120 \mathrm{ml}$ of fat were extracted using the Lipokit system (Medikan International, Seoul, Korea) and centrifuged using the Lipokit syringe to produce a total of $53 \mathrm{ml}$ of fat. After extracting $80 \mathrm{ml}$ of fat, collagenase was used to separate the fat and the stem cells. Subsequently, $50 \%$ of the isolated stem cells were mixed with the centrifuged fat and $38.2 \mathrm{ml}$ were transplanted into the right and $5.5 \mathrm{ml}$ into the left calf. The remaining

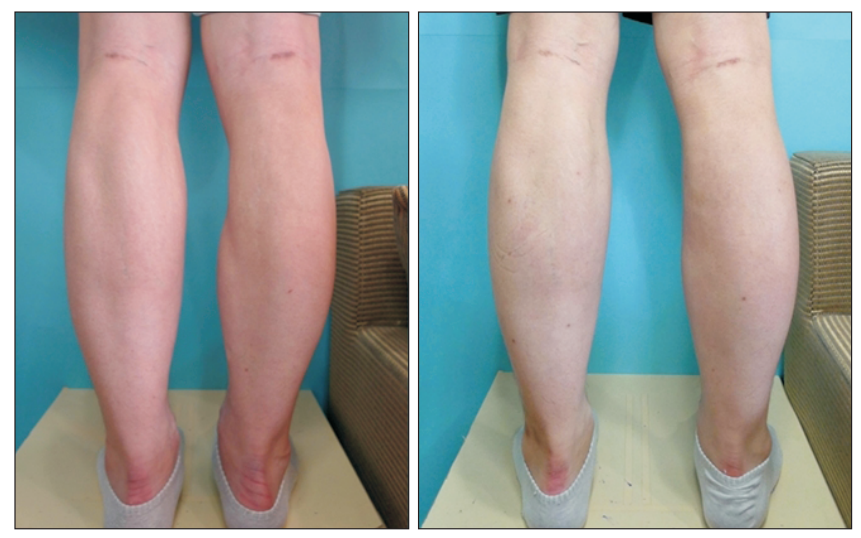

Fig. 1. Photographs obtained in a 35-year-old man in a standing position show: preoperative image (A) and image obtained 3 months postoperatively (B).
$50 \%$ of the isolated stem cells were injected using a $1 \mathrm{ml}$ syringe with a $25 \mathrm{G}$ needle (a total of $5 \mathrm{ml}$ ), directly into the contracted and shortened medial and lateral gastrocnemius muscles of both calves.

\section{Results}

Three months after the injection of adipose tissue derived stem cells (ADSC) and fat transplantation, photographs were obtained to evaluate the postoperative outcomes. The shortening of the right calf was corrected. The patient's right heel could now touch the ground, and his discomfort during walking was resolved. Fat transplantation led to near-complete correction of the dimple deformity of the calf on visual inspection. The calf muscle deformities were corrected using a soleus nerve block in the right calf and a medial gastrocnemius nerve block in the left calf (Fig. 1, 2).

\section{Discussion}

Selective neurectomy of the nerves innervating the calf muscles is useful to treat compensatory calf hypertrophy associated with calf reduction surgery [1]. Surgery can also be performed to directly remove the muscle. The procedure described in this report uses electrical stimulation to identify the nerve and apply high frequency waves to coagulate the nerve to a radius of within $5 \mathrm{~mm}$ at $95^{\circ} \mathrm{C}$ (AK-F50; Apro Korea, Gunpo, Korea). This procedure does not remove the muscle directly, and a 23G needle is utilized to minimize injury to the surrounding tissues. This procedure can be performed to treat the compensatory muscle hypertrophy that occurs secondary to calf nerve blocks and is useful to treat muscle deformities. Treatment for shortening
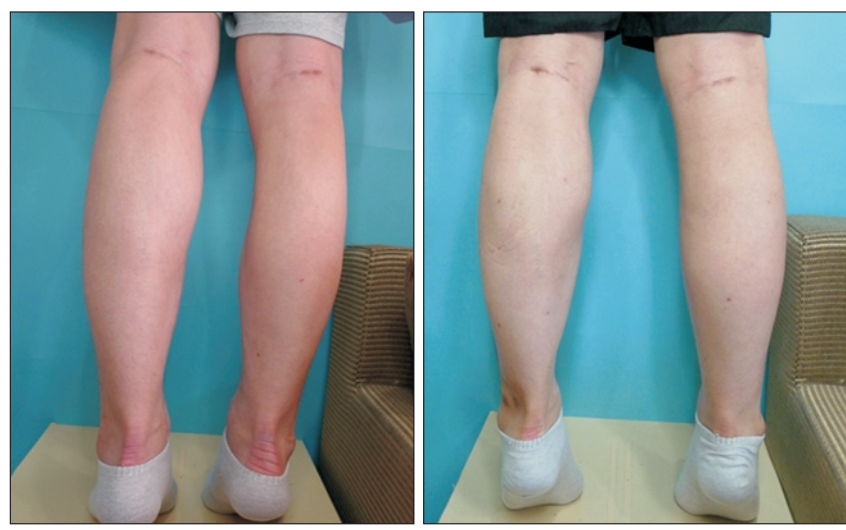

Fig. 2. Photographs obtained in a 35-year-old-man in a tip-toe position show: preoperative image (A) and image obtained 3 months postoperatively (B). 
and contraction of muscles caused by calf reduction and calf muscle resection surgeries must necessarily include a procedure that treats both, the fibrotic tissues in muscles and subcutaneous adhesions resulting from the surgery and liposuction, respectively. ADSC injections into the subcutaneous adhesions and fibrotic tissues are useful for this aforementioned purpose and can eventually improve the symptoms of muscle shortening and contraction caused by calf reduction and calf muscle resection surgeries. Additionally, adhesive depression of the skin and muscles secondary to the surgery can be effectively treated with fat transplantation using stem cells.

\section{Conclusion}

Calf cosmetic surgery can cause various types of contour deformities. Moreover, muscle contraction or shortening may occur after muscle resection surgery. Muscle resection surgery can cause muscle fibrosis and eventually causes dermal dimples and adhesions between the skin and the muscles. Restorative surgery can correct contour deformities of the calf, and compensatory muscle hypertrophy can be corrected by a selective nerve block procedure. Moreover, ADSC can be directly injected into the muscle cells to treat muscle contraction, and adhesive dimple deformities can be treated using fat transplantation with stem cells.

\section{Conflicts of interest}

The author has nothing to disclose.

\section{Reference}

1. Suh IS, Jung MS, Lee BH, Kim JH, Tak KS, Ahn DK. Classification and surgical correction of asymmetric calves in Asians. Plast Reconstr Surg 2014;134:707e-16e. 